Artigo de Revisão e/ou Atualização de Literatura

\title{
Órteses e próteses de membro superior impressas em 3D: uma revisão integrativa
}

\section{Upper limbs orthesis and prostheses printed in 3D: an integrative review}

\author{
Sandra Yoshie Uraga Morimoto ${ }^{a}$ (D), Ana Karina Pessoa da Silva Cabral ${ }^{a}$ (D), \\ Danielle Carneiro de Menezes Sanguinetti ${ }^{a}$ (D), Elvis da Silva Rodrigues de Freitas ${ }^{a}$ (D), \\ Giselle Schimidt Alves Diaz Merino ${ }^{\mathrm{b}}$ (D), José Ângelo Peixoto da Costa ${ }^{\mathrm{c}}$ (D), \\ Weldma Karlla Coelhod (D), Daniela Salgado Amaral ${ }^{\mathrm{b}}$ (D) \\ ${ }^{a}$ Universidade Federal de Pernambuco - UFPE, Recife, PE, Brasil. \\ ${ }^{\text {b} U n i v e r s i d a d e ~ F e d e r a l ~ d e ~ S a n t a ~ C a t a r i n a ~-~ U F S C, ~ F l o r i a n o ́ p o l i s, ~ S C, ~ B r a s i l . ~}$ \\ 'Instituto Federal de Pernambuco - IFPE, Recife, PE, Brasil. \\ ${ }^{\mathrm{d} C e n t r o ~ d e ~ R e a b i l i t a c ̧ a ̃ o ~ e ~ M e d i c i n a ~ F i ́ s i c a ~ P r o f e s s o r ~ R u y ~ N e v e s ~ B a p t i s t a ~-~ I M I P, ~ R e c i f e, ~ P E, ~ B r a s i l . ~}$
}

Como citar: Morimoto, S. Y. U., Cabral, A. K. P. S., Sanguinetti, D. C. M., Freitas, E. S. R., Merino, G. S. A. D., Costa, J. Â. P., Coelho, W. K., \& Amaral, D. S. (2021). Órteses e próteses de membro superior impressas em 3D: uma revisão integrativa. Cadernos Brasileiros de Terapia Ocupacional, 29, e2078. https://doi.org/10.1590/2526-8910.ctoAO2078

\begin{abstract}
Resumo
Introduçáo: A impressão tridimensional (3D) é capaz de confeccionar produtos físicos avançados e especializados por meio de tecnologia computadorizada e softwares específicos. Alguns desses produtos são as órteses e próteses, que podem favorecer a funcionalidade do sujeito em seu cotidiano. Objetivo: Identificar o tipo, o uso e a aplicabilidade da impressão 3D na confecção de órteses e próteses para membro superior. Método: Revisão integrativa realizada nas bases de dados PubMed, LILACS, Web of Science, Scopus e Science Direct, sem delimitação de tempo, na língua portuguesa, inglesa ou espanhola, seguindo os critérios de elegibilidade: estudos do tipo experimental, observacional e relatos de casos, cujo objeto de estudo foram as órteses e próteses impressas em 3D, com pacientes de qualquer idade e qualquer diagnóstico de comprometimento do membro superior. Resultados: Foram incluídos nove artigos, sete referentes ao uso da impressão 3D na confecção de prótese e dois referentes à confecção de órteses. Muitos dos estudos foram direcionados ao público infantil e os materiais mais utilizados para confecção foram o PLA e o ABS. A equipe multidisciplinar foi apresentada como fundamental no processo de avaliaçáo, criação e testagem dos dispositivos. Conclusáo: Apesar dos estudos analisados tangenciarem fases iniciais de desenvolvimento e investigaçáo da aplicabilidade da impressáo 3D na criaçáo de órteses e próteses, observou-se que já existem melhorias do custo-benefício gerado pelo uso desta tecnologia, bem como a possibilidade de gerar produtos mais versáteis. Apontando-se como um campo promissor para ampliar a aplicação da impressão 3D como recurso facilitador do processo de reabilitação.
\end{abstract}

Palavras-chave: Aparelhos Ortopédicos, Membros Artificiais, Impressão Tridimensional, Extremidade Superior, Reabilitação. 


\begin{abstract}
$\underline{\text { Abstract }}$
Introduction: Three-dimensional (3D) printing is capable of making advanced and specialized physical products using computerized technologies and specific software. Some of these products are orthosis and prostheses which can support patients' functionality in their daily lives. Objective: To identify the type, the usage, and the applicability of $3 \mathrm{D}$ printing on the development of upper limbs' orthosis and prostheses. Method: Integrative review carried out on the following databases: Pubmed, LILACS, Web of Science, Scopus e Science Direct, with no specific publication period, written in English, Spanish or Portuguese. It also had to fit the following criteria: experimental and observational studies and case reports which had $3 \mathrm{D}$ printed orthosis and prosthesis as the object of study with patients of any age or diagnosis of upper limb damage. Results: Nine studies were included. Seven referred to the use of 3D printing to make prosthesis and 2 to make orthosis. Many studies were directed to children and the most used materials were PLA and ABS. The multidisciplinary team was fundamental in the process of evaluation, creation, and testing of the devices. Conclusion: Despite the analyzed studies mention initial phases of development and investigation of the applicability of 3D printing in the creation of orthosis and prostheses, it was observed that cost-benefit improvements generated by the use of this technology already exist, as well as the possibility of generating more versatile products. It's a promising field to amplify the applicability of 3D printing as a resource facilitating the rehabilitation process.
\end{abstract}

Keywords: Orthotic Devices, Artificial Limbs, Printing; Three-Dimensional, Upper Extremity, Rehabilitation.

\title{
Introduçáo
}

A impressão tridimensional (3D), também já denominada de manufatura aditiva ou prototipagem rápida, caracteriza-se pela confecção de produtos físicos a partir de tecnologia computadorizada. Modelos virtuais tridimensionais desenvolvidos por softwares específicos são utilizados com o objetivo de dar maior liberdade de produção e design, principalmente com relação aos materiais e as formas variadas, a quem os confecciona (Gerstle et al., 2014; Maia, 2016).

Este tipo de tecnologia vem sendo desenvolvida há mais de 30 anos, porém, apenas na última década tomou maiores proporçóes e se tornou significativamente acessível, tanto para a populaçáo em geral como para aqueles que a estudam. Isso se deu devido ao seu grande alcance no que diz respeito à diversidade e ao baixo custo de produtos que podem ser confeccionados, valorizando sua exploraçáo por diversos mercados, inclusive o da saúde (Gerstle et al., 2014; Baronio et al., 2016; Maia, 2016).

$\mathrm{Na}$ área da saúde, a impressão em 3D vem ganhando espaço e sendo cada vez mais legitimada, já que é capaz de produzir agilmente protótipos mais avançados e especializados, como a impressão de órgáos específicos, de partes do corpo e de diferentes dispositivos de Tecnologia Assistiva (TA), por exemplo (Gerstle et al., 2014; Baronio et al., 2016).

Como nesta pesquisa abordamos as órteses e próteses impressas em 3D e estas compreendem recursos de Tecnologia Assistiva, temos que a TA pode ser definida como qualquer produto, equipamento, recurso, sistema de produtos, metodologias ou estratégias que visam restaurar e manter a funcionalidade de indivíduos com deficiências ou mobilidade reduzida e sua participação social (Brasil, 2009; Costa et al., 2015; Brasil, 2015a; Cook \& Polgar, 2015). 
A TA se classifica de acordo com áreas específicas, como: auxílios para a vida diária e vida prática; comunicação aumentativa e alternativa; recursos de informática e acessibilidade ao computador; projetos arquitetônicos para acessibilidade; auxílios de mobilidade; sistemas de controle de ambiente; adequação postural; auxílios para ampliação da função visual e recursos que traduzem conteúdos visuais em áudio ou informação tátil; auxílios para melhorar a função auditiva e recursos utilizados para traduzir os conteúdos de áudios em imagens, texto e língua de sinais; mobilidade em veículos; esporte e lazer; além de órteses e próteses (Brasil, 2009; Costa et al., 2015; Bersch, 2017).

Segundo a International Organization for Standardization (1989), a órtese, também denominada como dispositivo ortopédico, é definida como um dispositivo utilizado para modificar características estruturais e funcionais do sistema neuromusculoesquelético. Já as próteses, ou aparelhos protéticos, são definidas como dispositivos utilizados para substituir, totalmente ou em parte, um segmento do membro, ou parte do corpo humano, que seja ausente ou com deficiência.

Os dispositivos ortóticos e protéticos podem ser confeccionados e desenvolvidos a partir de diferentes materiais, selecionados e direcionados a cada sujeito, considerando fatores como condição financeira, nível de comprometimento da função e tipo de material mais adequado ao objetivo do dispositivo. Alguns materiais são: a madeira, o metal, a borracha, o couro e os polímeros de termoplástico, que variam de acordo com suas propriedades e constituiçóes (Agnelli \& Toyoda, 2003; Gonçalves \& Francisco, 2011; Maia, 2016). Nos dias atuais, é possível produzir dispositivos sofisticados e ajustáveis que permitem movimentos mais refinados e complexos a partir de tecnologias inovadoras, como a impressão tridimensional (Baronio et al., 2016; Maia, 2016).

As órteses e próteses são alguns dos recursos que visam promover a habilitação e reabilitação de pacientes com comprometimento físico que tenham implicaçóes na funcionalidade. Dessa forma, a utilização desses recursos auxilia no processo de tratamento dos pacientes, favorecimento da recuperação de suas funções orgânicas e contribuição para o melhor prognóstico do comprometimento em questão (Agnelli \& Toyoda, 2003; Cavalcanti \& Galvão, 2011; Radomski \& Latham, 2013).

A confecçáo de órteses e próteses impressas em $3 \mathrm{D}$ vem se tornando ainda mais forte, o que aumenta a visibilidade pelos profissionais da saúde e necessita cada vez mais de estudos para aprofundar o conhecimento de sua utilização. A impressão 3D permite a confecção desses produtos com altos níveis de customizaçáo, o que pode garantir aos pacientes um retorno mais brando e confortável à sua rotina (Gerstle et al., 2014; Baronio et al., 2016). Nessa perspectiva, o intuito deste estudo foi agrupar e sintetizar as pesquisas que estáo sendo realizadas ao redor do mundo, a respeito da confecção de órteses e próteses para membros superiores impressos em 3D, a fim de analisá-los, descrevê-los e proporcionar um avanço clínico e científico para a área. Deste modo, o estudo tem por objetivo identificar o tipo, o uso e a aplicabilidade da impressão 3D na confecção de órteses e próteses para membro superior.

\section{Método}

Trata-se de uma revisão integrativa, método bastante utilizado atualmente, visando à prática baseada em evidências. Este tipo de revisão é capaz de identificar, estudar e analisar criticamente dados resultantes de diversas pesquisas, gerando uma síntese das publicaçóes realizadas acerca de um tema e proporcionando a fundamentação de um estudo significativo (Souza et al., 2010). 
A coleta foi realizada em agosto de 2018, a partir do acesso às bases de dados PubMed, LILACS, Web of Science, Scopus e Science Direct, via rede da Comunidade Acadêmica Federada (CAFe) da Universidade Federal de Pernambuco, que é um serviço mantido pela Rede Nacional de Pesquisa e oferece facilidade de acesso ao conteúdo do Portal de Periódicos da CAPES. Os termos utilizados para a pesquisa foram cruzados utilizando os operadores booleanos "AND" e "OR". Para as bases de dados Scopus e Web of Science foram cruzados todos os descritores. No caso da Science Direct foram empregadas pesquisas isoladas apenas para os termos relativos à impressáo 3D, e nas bases de dados PubMed e LILACS foram utilizados os descritores Mesh/Decs (em negrito).

\section{Órtese}

1. (Orthotic devices or splints or orthosis or custom orthosis)

2. (Printing, three-dimensional or computer-aided design or rapid prototyping or additive manufacturing or computer-aided drafting or computer-aided manufacturing or threedimensional design or three-dimensional)

\section{3. (Rehabilitation)}

4. \#1 and \#2 and \#3

\section{Prótese}

1. (Artificial limbs or prostheses)

2. (Printing, three-dimensional or computer-aided design or rapid prototyping or additive manufacturing or computer-aided drafting or computer-aided manufacturing or threedimensional design or three-dimensional)

\section{3. (Rehabilitation)}

4. \#1 and \#2 and \#3

Foram incluídos artigos em língua portuguesa, inglesa e espanhola, com desenhos metodológicos do tipo experimental, observacional e relatos de casos, que tinham como objeto de estudo órteses e próteses impressas em 3D e que o produto fosse destinado a pacientes com afecçóes nos membros superiores, independente do diagnóstico e/ou idade. Trabalhos que discutiam acerca de outros produtos em $3 \mathrm{D}$, que não órteses e próteses de membro superior, foram excluídos. Não foi delimitado o período de publicação dos artigos por se tratar de um componente recente na área da saúde.

Os artigos foram selecionados a partir de dois revisores (revisor A e B), mediante leitura inicial dos títulos, resumos e textos na íntegra, utilizando-se os critérios de elegibilidade estabelecidos. Posteriormente, as amostras finais de cada autor foram discutidas entre estes a fim de se chegar a um consenso entre os textos divergentes apresentados na coleta de cada um. Um terceiro autor, que permaneceu imparcial, foi necessário, a fim de verificar os questionamentos levantados acerca dos textos divergentes entre cada autor da análise por pares e, por fim, julgar os resultados concernentes à pesquisa. Ao final, foram analisadas todas as referências dos textos selecionados e aquelas que respondiam aos critérios de elegibilidade foram incluídas. Todos os autores responsáveis pela análise dos artigos coletados são profissionais ou estudantes de saúde, das áreas de Terapia Ocupacional e Fisioterapia. 
Os artigos incluídos foram analisados e extraídos dos mesmos locais das informaçóes pertinentes para a caracterizaçáo destes, como o desenho do estudo, população do estudo, tipos de dispositivos estudados, local onde foram realizados e profissionais envolvidos (Tabela 1 e 2). Ademais, foi possível explanar, por meio da Tabela 3, a seguir, o panorama geral acerca de cada um dos textos quanto aos seus objetivos, principais resultados, limitaçóes e sugestóes. Por fim, na Tabela 4, a seguir, foi explicitado o detalhamento do desenvolvimento dos dispositivos, trazendo aspectos do processo de desenvolvimento, dos métodos avaliativos e reavaliativos dos estudos, bem como os custos apresentados por cada um. Os resultados foram apresentados de forma descritiva e discutidos com base nas inferências contidas na literatura até a explicitação das conclusôes dos autores.

\section{Resultados}

Com a busca na literatura e as consideraçóes dos critérios de elegibilidade, obteve-se o total das cinco bases de dados utilizadas e uma amostra de 9 artigos que foram analisados nesta revisão. Os resultados completos estáo apresentados na Figura 1.

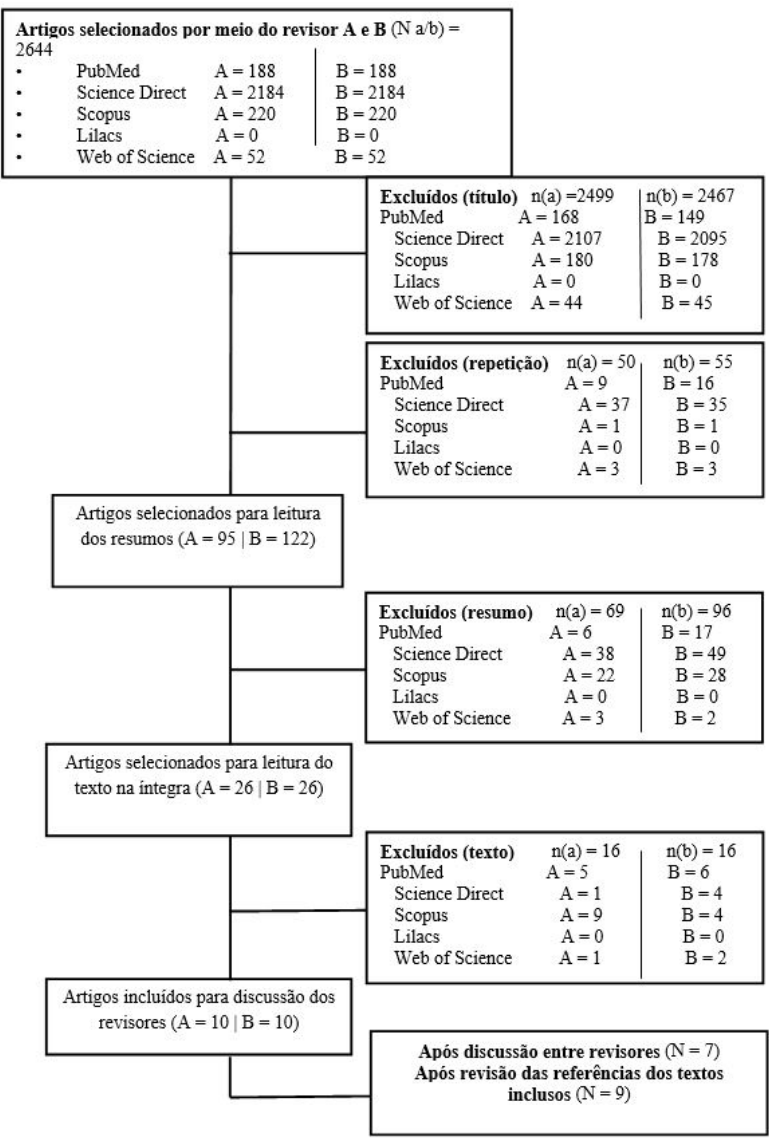

Figura 1. Etapas de análise para elaboração da amostra final.

Os 9 artigos incluídos estão apresentados na Tabela 1, organizados em ordem cronológica de publicação, assim como em todas as tabelas seguintes. 
Tabela 1. Artigos inclusos.

\begin{tabular}{ccc}
\hline AUTOR (ANO) & Local & TíTULO \\
\hline Yoshikawa et al. (2013) & Japão & Trans-radial prosthesis with three opposed fingers \\
\hline Zuniga et al. (2015) & EUA & $\begin{array}{c}\text { Cyborg beast: a low-cost 3d-printed prosthetic hand for children with upper-limb } \\
\text { differences }\end{array}$ \\
\hline Yoshikawa et al. (2015) & Japão & Rehand: realistic electric prosthetic hand created with 3D printer \\
\hline Gretsch et al. (2016) & EUA & Development of novel 3D printed robotic prosthetic for transradial amputees \\
\hline Zuniga et al. (2017) & EUA & The development of a low-cost three-dimensional printed shoulder, arm and hand \\
prostheses for children
\end{tabular}

Os artigos analisados foram publicados nos últimos 5 anos, exceto o estudo de Yoshikawa et al., publicado no ano de 2013. Os locais onde estão explorando a impressão 3D como meio de confecção das órteses e próteses variaram entre Estados Unidos da América, Japão, Brasil, China e México, sendo todos realizados no país referido e publicados em meios internacionais, tendo como referência o Brasil (Tabela 1). Na Tabela 2, tem-se que as próteses foram mais estudadas do que as órteses, aparecendo em 7 dos 9 artigos. Dentre essas categorias, as órteses dinâmicas e próteses mioelétricas foram as mais abordadas. Além disso, verificou-se que a maior parte dos estudos de desenvolvimento protético diz respeito ao público infantil.

De forma geral, os trabalhos apresentaram participação de mais de uma categoria profissional, muitas vezes incluindo os profissionais da área da saúde. As categorias citadas foram: protesista, que realizou avaliaçóes antropométricas, e terapeuta ocupacional, que ficou responsável pela avaliação de funcionalidade; médicos, para a consulta médica e avaliaçáo do paciente; fisioterapeuta, para o treino de reabilitaçáo e preparo para o uso da prótese; e orientador técnico e designer, para a confecção dos produtos. Porém, em alguns estudos não foram especificadas as categorias e foram identificados como equipe multidisciplinar (Tabela 2).

Tabela 2. Caracterização dos estudos.

\begin{tabular}{|c|c|c|c|c|}
\hline Autor (ano) & $\begin{array}{l}\text { Desenho do } \\
\text { Estudo }\end{array}$ & Populaçáo do estudo & Tipo de dispositivo & Profissionais envolvidos \\
\hline $\begin{array}{l}\text { Yoshikawa et al. } \\
\qquad(2013)\end{array}$ & NI & $\begin{array}{l}\text { Paciente de } 60 \text { anos com } \\
\text { amputação transradial }\end{array}$ & Prótese elétrica & NI \\
\hline Zuniga et al. (2015) & NI & Crianças com membro reduzido & Prótese & Protesista e terapeuta ocupacional \\
\hline $\begin{array}{l}\text { Yoshikawa et al. } \\
(2015)\end{array}$ & NI & Amputados do antebraço & Prótese mioelétrica & NI \\
\hline Gretsch et al. (2016) & Relato de caso & $\begin{array}{l}\text { Paciente do sexo feminino, } 13 \\
\text { anos, com amputação } \\
\text { transradial }\end{array}$ & Prótese mioelétrica & Médico, orientador técnico \\
\hline Zuniga et al. (2017) & Nota técnica & $\begin{array}{c}\text { Criança com amputaçáo de } \\
\text { ombro }\end{array}$ & Prótese mioelétrica & NI \\
\hline Xu et al. (2017) & Relato de caso & Criança amputada & Prótese & $\begin{array}{l}\text { Equipe multidisciplinar de saúde e } \\
\text { pais (cuidadores) }\end{array}$ \\
\hline Silva et al. (2018) & Relato de Caso & $\begin{array}{l}\text { Criança com amputação } \\
\text { transtibial bilateral }\end{array}$ & Prótese mecânica & $\begin{array}{c}\text { Terapeuta ocupacional, protesista } \\
\text { e designer }\end{array}$ \\
\hline $\begin{array}{l}\text { Merchant et al. } \\
\text { (2018) }\end{array}$ & NI & $\begin{array}{l}\text { Pessoas com algum tipo de lesão } \\
\text { medular }\end{array}$ & $\begin{array}{l}\text { Órtese dinâmica com } \\
\text { componente de robótica }\end{array}$ & NI \\
\hline $\begin{array}{l}\text { García-García et al. } \\
\text { (2018) }\end{array}$ & Relato de caso & $\begin{array}{c}\text { Paciente de } 60 \text { anos com } \\
\text { dificuldade na amplitude de } \\
\text { movimento }\end{array}$ & Órtese dinâmica & Equipe multidisciplinar \\
\hline
\end{tabular}

NI: Não Informado. 
Foi observado que, em todos os estudos que atenderam o critério de inclusão, um dos principais objetivos do uso da impressáo 3D para a confecção de órteses e próteses foi o baixo custo gerado por esta, além da maior precisão no desenvolvimento das peças e produtos. Ainda nessa perspectiva, apesar de todos terem obtido resultados positivos após o uso e teste dos produtos finais, algumas limitaçóes foram levantadas, como a baixa força de preensão e a dificuldade na realização de movimento de motricidade fina, para o caso das próteses, por exemplo, e a dificuldade relacionada ao alto consumo de tempo na aquisição de imagens da anatomia da máo e posterior impressão do modelo 3D, para o caso da órtese (Tabela 3). Quanto aos materiais mais utilizados para a confecção dos produtos, encontramos dois principais: o Ácido Polilático (PLA), utilizado em 5 dos 9 artigos, e a Acrilonitrila Butadieno Estireno (ABS), utilizado em 6 dos 9 artigos, sendo que, em alguns destes estudos, foram utilizados ambos os materiais.

Tabela 3. Panorama geral dos artigos inclusos.

\begin{tabular}{|c|c|c|c|c|}
\hline Autor (ano) & Objetivo & Principais resultados & Limitaçóes & Sugestóes \\
\hline $\begin{array}{l}\text { Yoshikawa et al. } \\
\text { (2013) }\end{array}$ & $\begin{array}{l}\text { Projetar e desenvolver uma } \\
\text { prótese transradial leve e de } \\
\text { baixo custo. }\end{array}$ & $\begin{array}{l}\text { Participante conseguiu vestir } \\
\text { sozinho a prótese, agarrar objetos } \\
\text { abstratos e operar intuitivamente } \\
\text { a prótese. Soquete suportou o } \\
\text { coto curto. }\end{array}$ & NI & $\begin{array}{l}\text { Melhorar a rigidez da máo e } \\
\text { avaliar a usabilidade, } \\
\text { versatilidade e durabilidade } \\
\text { da prótese em mais } \\
\text { amputados. }\end{array}$ \\
\hline $\begin{array}{l}\text { Zuniga et al. } \\
\text { (2015) }\end{array}$ & $\begin{array}{l}\text { Descrever uma prótese de } \\
\text { baixo custo tridimensional } \\
\text { impressa para crianças }\end{array}$ & $\begin{array}{l}\text { A prótese foi positiva na QV e na } \\
\text { AVD. Teve baixo custo e boa } \\
\text { capacidade de ajuste. }\end{array}$ & NI & $\begin{array}{c}\text { Novos estudos para } \\
\text { examinar a funcionalidade, } \\
\text { validade, durabilidade, } \\
\text { benefícios e taxa de rejeiçăo. }\end{array}$ \\
\hline $\begin{array}{l}\text { Yoshikawa et al. } \\
\text { (2015) }\end{array}$ & $\begin{array}{l}\text { Relatar a Rehand, uma } \\
\text { prótese elétrica realística. }\end{array}$ & $\begin{array}{c}\mathrm{O} \text { Rehand fornece a função } \\
\text { básica de preensão nas AVD } \\
\text { com amputados do antebraço. }\end{array}$ & $\begin{array}{l}\text { Mal desempenho na } \\
\text { manipulaçáo de objetos } \\
\text { pesados e nos } \\
\text { movimentos finos. }\end{array}$ & $\begin{array}{l}\text { Melhorar a rigidez da mão e } \\
\text { o tamanho da caixa de } \\
\text { controle. Avaliar a máo com } \\
\text { mais amputados nas AVD. }\end{array}$ \\
\hline $\begin{array}{l}\text { Gretsch et al. } \\
\text { (2016) }\end{array}$ & $\begin{array}{l}\text { Desenvolver um braço } \\
\text { protético de baixo custo para } \\
\text { abordar as limitaçóes da } \\
\text { Robohand. }\end{array}$ & $\begin{array}{l}\text { Produto com movimento de } \\
\text { oposiçáa do polegar, preensáo } \\
\text { com os cinco dedos, baixo peso e } \\
\text { baixo custo. }\end{array}$ & $\begin{array}{l}\text { Limitaçóes da bateria, } \\
\text { durabilidade, força de } \\
\text { preensão e ruídos. }\end{array}$ & $\begin{array}{l}\text { Desenvolvimento de } \\
\text { melhorias no protótipo. }\end{array}$ \\
\hline $\begin{array}{l}\text { Zuniga et al. } \\
\text { (2017) }\end{array}$ & $\begin{array}{l}\text { Projetar uma prótese de } \\
\text { ombro de baixo custo para } \\
\text { atividades bimanuais e } \\
\text { unilaterais. }\end{array}$ & $\begin{array}{c}\text { O dispositivo permitiu melhora } \\
\text { no desvio de coluna, equilibrio e } \\
\text { desempenho em atividades } \\
\text { bimanuais. }\end{array}$ & $\begin{array}{l}\text { Baixa força de preensão } \\
\text { e baixa durabilidade do } \\
\text { dispositivo. }\end{array}$ & $\begin{array}{c}\text { Melhorar o funcionamento } \\
\text { das articulaçóes MCF e a } \\
\text { tecnologia de impressáo 3D. }\end{array}$ \\
\hline Xu et al. (2017) & $\begin{array}{l}\text { Relatar o caso de uma } \\
\text { criança com uso de prótese } \\
\text { após a amputaçáo } \\
\text { traumática do punho. }\end{array}$ & $\begin{array}{l}\text { A prótese teve baixo custo, bom } \\
\text { ajuste e customizaçáo. Após o } \\
\text { treinamento e reabilitação, } \\
\text { observou-se melhora da } \\
\text { funcionalidade. }\end{array}$ & $\begin{array}{l}\text { A prótese nâo é aplicável } \\
\text { para alguns movimentos } \\
\text { finos e bimanuais. }\end{array}$ & $\begin{array}{l}\text { Comparar a longo prazo } \\
\text { próteses em 3D com } \\
\text { próteses convencionais e } \\
\text { realizar treinos mais } \\
\text { avançados. }\end{array}$ \\
\hline Silva et al. (2018) & $\begin{array}{c}\text { Descrever o } \\
\text { desenvolvimento de uma } \\
\text { prótese mecânica transradial } \\
\text { para uma criança com } \\
\text { amputaçáa bilateral do MS. }\end{array}$ & $\begin{array}{l}\text { Boa durabilidade e fixaçáo da } \\
\text { prótese. Dispositivo leve e que } \\
\text { não demanda muito esforço. } \\
\text { Confortável, fácil de ajustar e } \\
\text { higienizar. }\end{array}$ & $\begin{array}{l}\text { Dificuldade na } \\
\text { motricidade fina, como } \\
\text { escrever (solucionado } \\
\text { com o uso de } \\
\text { adaptador) e fechar } \\
\text { zíper. }\end{array}$ & NI \\
\hline $\begin{array}{l}\text { Merchant et al. } \\
\text { (2018) }\end{array}$ & $\begin{array}{c}\text { Desenvolver protótipo de } \\
\text { um sistema ortótico para ser } \\
\text { usado na reabilitaçáa do MS. }\end{array}$ & $\begin{array}{l}\text { O dispositivo pode ser ajustado a } \\
\text { diferentes usuários e facilmente } \\
\text { transportado. Permite ajuste para } \\
\text { diferentes movimentos. }\end{array}$ & NI & NI \\
\hline $\begin{array}{l}\text { García- } \\
\text { García et al. } \\
\text { (2018) }\end{array}$ & $\begin{array}{l}\text { Propor uma metodologia } \\
\text { baseada em engenharia } \\
\text { reversa para o } \\
\text { desenvolvimento de órteses } \\
\text { progressivas dinâmicas ou } \\
\text { estáticas para a mão. }\end{array}$ & $\begin{array}{c}\text { Alto nível de aceitação na } \\
\text { reabilitaçáo, especialmente para } \\
\text { pacientes com AVC agudo. O } \\
\text { método de engenharia reversa } \\
\text { oferece alto grau de } \\
\text { personalizaçáo de órteses. }\end{array}$ & $\begin{array}{c}\text { Houve um custo alto de } \\
\text { tempo na aquisiçáo de } \\
\text { imagens da anatomia da } \\
\text { máo. }\end{array}$ & $\begin{array}{c}\text { Realização de ensaios clínicos } \\
\text { para mais evidências. Tornar } \\
\text { o dispositivo mais leve e } \\
\text { permitir que a pele respire, } \\
\text { melhorando sua utilizaçáo. }\end{array}$ \\
\hline
\end{tabular}

NI: Não informado. MS: Membro Superior. MCF: Metacarpofalangeana. AVD: Atividades de Vida Diária. QV: Qualidade de Vida. AVC: Acidente Vascular Cerebral. 
No que se refere ao valor do produto, foi possível observar que as próteses variaram entre $\$ 20$ e \$1250 dólares, dependendo do público e do tipo de prótese. Já os valores referentes aos custos dos materiais das órteses não foram disponibilizados pelos estudos. Além disso, no que se refere ao desenvolvimento dos produtos, dentre os nove artigos, seis apresentaram o processo de avaliação, ideação e confecção do dispositivo, entretanto, elaborados de maneira distintas. Ademais, identificou-se que apenas um dos estudos apresenta a realização de reabilitação física e treino de usos específicos para utilização dos dispositivos confeccionados, e cinco estudos realizaram algum tipo de teste de funcionalidade dos produtos desenvolvidos. Os testes empregados foram: Southampton Hand Assessment Procedure (SHAP), que verifica a eficácia da prótese por meio da análise dos padróes de preensão e sua frequência de uso nas atividades de vida diária; The Children Amputee Prosthetics Projects (CAPP) Score e The University of New Brunswick Test of Prosthetic Function for Unilateral Amputee (UNB test), que avalia a funcionalidade diária da prótese; avaliação observacional pela terapeuta da realização de atividades em que a paciente apresentou maior dificuldade; e simulação de performance do dispositivo para cada articulaçáo envolvida durante a terapia.

Tabela 4. Detalhamento do desenvolvimento dos dispositivos.

\begin{tabular}{|c|c|c|c|c|}
\hline Textos & Processo de Desenvolvimento & Avaliação & Reavaliação & Custo total \\
\hline $\begin{array}{l}\text { Yoshikawa et al. } \\
\qquad(2013)\end{array}$ & $\mathrm{NI}$ & $\begin{array}{l}\text { Prótese para amputação } \\
\text { transradial ajustável }\end{array}$ & SHAP & $<\$ 400$ \\
\hline $\begin{array}{l}\text { Zuniga et al. } \\
\qquad(2015)\end{array}$ & $\begin{array}{l}\text { Avaliação - calibração da escala } \\
\text { métrica - utilização de gráfico para } \\
\text { estimação do tamanho da máo } \\
\text { protética - confecção. }\end{array}$ & $\begin{array}{c}\text { Medidas antropométricas } \\
\text { do MS }\end{array}$ & NI & $\$ 50$ \\
\hline $\begin{array}{l}\text { Yoshikawa et al. } \\
\qquad(2015)\end{array}$ & $\begin{array}{l}\text { Avaliação - mecanismo de agarrar foi } \\
\text { projetado em um software CAD 3D } \\
\text { - impressão 3D - encaixe das partes }\end{array}$ & $\begin{array}{l}\text { Baseado em um modelo } \\
\text { protético comercial } \\
\text { (escâner) }\end{array}$ & SHAP & $<\$ 1250$ \\
\hline $\begin{array}{l}\text { Gretsch et al. } \\
\qquad(2016)\end{array}$ & $\begin{array}{c}\text { Avaliação - impressão - } \\
\text { componentes eletrônicos - } \\
\text { programação do sistema arduino }\end{array}$ & $\begin{array}{l}\text { Mensuração do membro } \\
\text { afetado e não afetado }\end{array}$ & $\begin{array}{l}\text { Relato do paciente e seus } \\
\text { familiares após o uso }\end{array}$ & $+/-\$ 300$ \\
\hline $\begin{array}{l}\text { Zuniga et al. } \\
\quad(2017)\end{array}$ & $\mathrm{NI}$ & Questionário médico & $\begin{array}{l}\text { Relato do paciente e seus } \\
\text { familiares após o uso }\end{array}$ & $\$ 200$ \\
\hline Xu et al. (2017) & $\begin{array}{l}\text { Avaliação - escaneamento - } \\
\text { customização do modelo - impressão } \\
\text { dos componentes da prótese - } \\
\text { junção dos componentes }\end{array}$ & $\begin{array}{l}\text { Questionário clínico; } \\
\text { design de código aberto; } \\
\text { mensuração dos membros } \\
\text { afetado e náo afetado + } \\
\text { escaneamento }\end{array}$ & $\begin{array}{l}\text { CAPP Score e UNB test } \\
\text { após acompanhamento, } \\
\text { treino de uso e } \\
\text { reabilitação (pré-protético } \\
\text { e pós protético) }\end{array}$ & $\$ 20$ \\
\hline $\begin{array}{l}\text { Silva et al. } \\
\quad(2018)\end{array}$ & $\begin{array}{l}\text { Avaliação - escolha do melhor tipo } \\
\text { de prótese - processo de } \\
\text { prototipagem e design - testagem } \\
\text { protética - concepção final do } \\
\text { produto - teste final }\end{array}$ & $\begin{array}{l}\text { Questionário clínico e } \\
\text { funcional; mensuração do } \\
\text { molde para o membro; } \\
\text { escaneamento }\end{array}$ & $\begin{array}{l}\text { Avaliação observacional de } \\
\text { atividades pela terapeuta }\end{array}$ & NI \\
\hline $\begin{array}{l}\text { Merchant et al. } \\
\text { (2018) }\end{array}$ & $\begin{array}{l}\text { Avaliação - seçóes mecânicas da } \\
\text { órtese foram modeladas no software } \\
\text { CAD - transferência do modelo } \\
\text { mecânico para a interface Simulink- } \\
\text { MATLAB - execução do } \\
\text { controlador em placa digital }\end{array}$ & $\begin{array}{c}\text { Análise mecânica dos } \\
\text { componentes necessários } \\
\text { para executar os } \\
\text { movimentos do braço }\end{array}$ & $\begin{array}{c}\text { Simulação e } \\
\text { implementação real em } \\
\text { um sujeito }\end{array}$ & NI \\
\hline $\begin{array}{l}\text { García- } \\
\text { García et al. } \\
(2018)\end{array}$ & $\begin{array}{c}\text { Avaliação - identificação dos } \\
\text { elementos da órtese - aquisição de } \\
\text { imagens do membro por MRI - } \\
\text { escaneamento para geração do } \\
\text { modelo - impressão }\end{array}$ & $\begin{array}{l}\text { Questionário clínico a fim } \\
\text { de entender as demandas } \\
\text { do paciente e melhor tipo } \\
\text { de tratamento }\end{array}$ & NI & NI \\
\hline
\end{tabular}

NI: Não informado. MS: Membro Superior. SHAP: Southampton Hand Assessment Procedure. CAPP: The Children Amputee Prosthetics Projects. UNB test: The University of New Brunswick Test of Prosthetic Function for Unilateral Amputee. CAD: Computer-assisted Design. MRI: Magnetic Resonance Imaging. 


\section{Discussão}

A impressão 3D, ou manufatura aditiva, vem crescendo mundialmente em termos de uso e pesquisa científica, inclusive no âmbito da saúde (Guerra Neto et al., 2018). No entanto, esta revisão integrativa, realizada com o recorte nas órteses e próteses para membros superiores, demonstra que ainda são poucos os estudos e práticas que envolvem o uso da impressão 3D nesta área, considerando as bases utilizadas. Os artigos analisados tratam de trabalhos recentes, ainda em fases iniciais de teste e aperfeiçoamento metodológico, nos quais foi observada uma predominância no desenvolvimento das próteses, sendo as órteses menos frequentes.

Em uma revisão sistemática realizada por Diment et al. (2018) sobre prótese, também foram encontrados estudos com baixos níveis de evidência, sendo apenas um destes forte $o$ suficiente para demonstrar um efeito clinicamente significativo. Outra revisão sistemática realizada por Guerra Neto et al. (2018) sobre órteses e próteses impressas em 3D aponta que, com essa tecnologia, esses dispositivos podem estabelecer melhores custos-benefícios.

Próteses são importantes recursos empregados no âmbito da reabilitação, já que se caracterizam pela substituição de algum membro perdido ou com má formação (Polis, 2009; Cavalcanti \& Galvão, 2011). Podem ser classificadas em diferentes tipos e modelos de aparelhagem, como as próteses estáticas, ativas e mioelétricas (Polis, 2009).

As próteses mioelétricas foram as mais frequentes no estudo em questão. Segundo Radomski \& Latham (2013) e Pereira (2016), estas são dispositivos que se utilizam dos sinais eletromiográficos (EMG), captados através de eletrodos que ficam em contato com a pele para a amplificação da contração muscular em um membro residual. Essa prevalência sugere a possibilidade de que isto ocorreu devido ao seu maior nível de complexidade de confecçáo, pela necessidade da integraçáo com sistemas eletromiográficos, quando comparada com os demais modelos protéticos. Assim, a partir da impressão 3D, esses dispositivos podem ser confeccionados, gerando modelos mais versáteis, ou seja, mais especializados e aperfeiçoados, que permitem a realização de movimentos mais precisos e refinados (Polis, 2009; Maia, 2016).

Dentre os nove estudos incluídos, cinco trouxeram como foco o desenvolvimento de próteses para crianças. Resultado semelhante é encontrado no estudo de Diment et al. (2018), em que a predominância foi esse público. É nesse período da vida em que o processo de crescimento e desenvolvimento ocorrem de maneira mais rápida, levando a diversas alteraçóes no corpo do indivíduo, o que torna inconcebível a possibilidade de acompanhamento da produçáo de uma nova prótese em resposta a este crescimento em cada uma de suas etapas (Krebs et al., 1991). Devido a isso, é justificável que muitos estudos estejam explorando o desenvolvimento desses dispositivos, a partir da tecnologia de impressão $3 \mathrm{D}$, tendo em vista que esta permite maior rapidez e menor custo em sua produção.

No que se refere às órteses, Gonçalves \& Francisco (2011) explicam que é todo e qualquer dispositivo que pode ser aplicado para uso externo ao corpo. Apesar de se apresentarem sob diversos modelos e funcionalidades, possuem como principais objetivos: proteger uma regiáo inflamada com adequado posicionamento, garantir a estabilidade de determinado segmento corporal, restaurar e reabilitar movimentos perdidos ou comprometidos, prevenir contraturas e modificar o tônus muscular. Estas podem se diferenciar em estáticas ou dinâmicas, moldadas ao corpo ou pré-fabricadas (Gonçalves \& Francisco, 2011; Radomski \& Latham, 2013; Guerra Neto et al., 2018). No estudo, as órteses apresentadas foram do tipo dinâmicas, que se caracterizam pela aplicação de uma 
força suave e intermitente para a movimentação de uma articulação específica em sua angulação correta, a partir do alongamento dos tecidos, e que permitem, dessa forma, que esses movimentos sejam realizados de maneira mais funcional, restringindo padróes musculares inadequados (Radomski \& Latham, 2013).

Apenas 2, dentre os 9 artigos incluídos, tinham a órtese como objeto de estudo, assim, diferentes questóes podem ser levantadas. Uma delas é que, apesar do auxílio do escâner $3 \mathrm{D}$ que permite a aquisição de modelos anatômicos virtuais tridimensionais do membro para impressão, é possível que ainda exista uma deficiência desta tecnologia na viabilidade de acabamentos e ajustes finais, que normalmente são realizados no próprio membro do indivíduo para melhor encaixe da órtese no membro. Outra questão é quanto aos custos para confecção e aquisição desses dispositivos. Por se caracterizarem como produtos mais acessíveis à população, em termos de custo, diferentemente das próteses, são menos exploradas no âmbito da impressão $3 \mathrm{D}$ e, quando o são, estão relacionadas às órteses dinâmicas que, dentre os tipos de órtese, são os modelos que exigem maior nível de complexidade na confecção e custos um pouco mais altos.

Observando o processo de desenvolvimento dos produtos impressos em 3D encontrados, foram identificados nos artigos a indicaçáo de limitaçóes que podem ter interferido nos resultados. Tendo em vista que a maior parte dos estudos abordaram a confecção de próteses, observou-se com maior frequência dificuldades quanto ao uso destes dispositivos, sendo o principal deles a baixa força de preensão e limitaçóes para a realização de atividades que demandam maior destreza manual. Essa última ainda se apresenta como um grande desafio para as próteses de membro superior tradicionais, já que não permitem, em sua maioria, o reconhecimento do toque de maneira fidedigna como a realizada pelos mecanorreceptores de uma mão humana (Martins, 2018). Apesar disso, grande parte destes estudos não apresentaram sugestōes específicas para essas dificuldades, relatando apenas a necessidade da realização de estudos mais aprofundados na área, a fim de gerar alternativas para estes problemas.

Outra limitação relatada foi a necessidade de realização de estudos com maiores níveis de evidência, que possam comprovar a eficiência da utilização da impressão 3D no desenvolvimento de produtos, como órteses e próteses, o que ratifica que as pesquisas ainda são recentes a respeito do tema e permeiam metodologias com níveis de evidência mais frágeis. Assim, ensaios clínicos, caracterizados como os estudos que melhor comprovam a eficácia de um tratamento ou intervenção (Nedel \& Silveira, 2016), e estudos de usabilidade, que correspondem a testes de ergonomia e design de um produto (Paschoarelli \& Menezes, 2009), podem ser possíveis tipos de pesquisas que contribuirão para o crescimento científico do uso da impressão 3D de órteses e próteses de membros superiores.

Uma equipe multidisciplinar é fundamental no processo de desenvolvimento do produto, seja ele ortótico ou protético, já que cada profissional executa um papel diferente que vai contribuir cooperativamente para o resultado final, como também, consideram os diversos aspectos englobados pelo processo de avaliaçáo e confecção dos produtos. Diversos estudos já abordam a importância da implementação e manutenção da comunicação desta equipe com objetivo de atingir os mais altos níveis de customização e atender as demandas específicas de cada sujeito (Baronio et al., 2016; Rodrigues Júnior et al., 2018; Wagner et al., 2018).

A equipe é composta por profissionais capacitados para manejo da mecânica e técnicas utilizadas para o funcionamento adequado da tecnologia, como os engenheiros, designers, arquitetos e protesistas, e também por profissionais da área da saúde que são fundamentais por compreenderem mais profundamente as demandas clínicas, biológicas, anatômicas e 
de funcionalidade e participação social do sujeito, a fim de proporcionar maior conforto, segurança e facilidade no uso do dispositivo (Bersch, 2017).

Apesar da maioria dos artigos terem citado profissionais de saúde em seus estudos, apenas Xu et al. (2017) indicaram a realização de um treino de uso específico e reabilitaçáa por fisioterapeuta. O treino foi capaz de facilitar o controle da prótese pelo sujeito por meio de movimentos repetitivos, além de contribuir para a realizaçáo de atividades do dia a dia. Apesar dos demais não terem em suas metodologias de estudo o processo de treino de uso do dispositivo, foi possível observar que 5, dentre os 9 trabalhos, realizaram algum tipo de avaliaçáo da funcionalidade do produto por meio de instrumentos padronizados ou por meio do terapeuta ocupacional, que se caracteriza pelo profissional responsável pela avaliação da funcionalidade e desempenho ocupacional das atividades de vida diária do sujeito, a fim de diminuir suas dificuldades funcionais nestas (Rodrigues Júnior et al., 2018).

Segundo a Resolução no 458, de 20 de novembro de 2015 (Brasil, 2015b), é de competência do terapeuta ocupacional atuar nas práticas e serviços de Tecnologia Assistiva, sendo as órteses e próteses uma de suas áreas de atuação profissional, identificando a necessidade do uso ou não de órteses e próteses, prescrever, desenvolver e confeccionar estes ou outros dispositivos de assistência e ajuda técnica, a fim de potencializar seu processo de tratamento, minimizar sequelas e melhorar o desempenho ocupacional da pessoa em seu cotidiano e, consequentemente, sua participação social. Ademais, foi observado, nesta pesquisa, que os estudos que demonstraram algum tipo de treino ou teste funcional dos indivíduos para verificar a eficiência dos dispositivos, de maneira geral, apresentaram menos limitaçóes em seus resultados. Além disso, um desses estudos foi capaz de solucionar o problema apenas com uma adaptação feita pelo terapeuta ocupacional (Silva et al., 2018).

Com relação aos materiais utilizados para impressão dos dispositivos, o PLA e o ABS foram os mais citados. Ambos são tipos de termoplástico bastante utilizados na prática da impressáo 3D atualmente, porém, com diferentes propriedades e características. O PLA é um material biodegradável, derivado de recursos naturais, como a mandioca, o milho e a cana de açúcar. É considerado muito rígido e resistente, ou seja, com pouca flexibilidade e baixa resistência à tração, o que dificulta o desenvolvimento de dispositivos que precisam de encaixes mais complexos e que exijam maior nível de complexidade do movimento (Aguiar \& Yonezawa, 2014; Mallmann, 2018).

Ademais, o PLA ainda possui diversas vantagens, como a sustentabilidade, atribuída aos recursos renováveis (Drumright et al., 2000), a biocompatibilidade, que previne efeitos tóxicos e carcinogênicos (Athanasiou et al., 1996), a processabilidade, devido ao melhor processamento térmico quando comparado com outros biopolímeros (Auras et al., 2004), e a economia de energia, quando comparado com polímeros derivados do petróleo (Vink et al., 2003), o que o torna também uma alternativa promissora no mercado. O ABS, por sua vez, é um material derivado do petróleo, o que pode torná-lo menos recomendado, já que, do ponto de vista ecológico, leva períodos mais longos de tempo para se decompor. Entretanto, dentre suas propriedades, apresenta maior flexibilidade, o que permite o desenvolvimento de protótipos que oferecem maior liberdade de movimento ao sujeito (Aguiar \& Yonezawa, 2014).

Nos estudos analisados, apesar do PLA e ABS não terem sido os únicos materiais utilizados, observou-se que os custos destes materiais, de forma geral, foram reduzidos. Entretanto, esses valores ainda são muito variáveis entre uma pesquisa e outra. Segundo McGimpsey \& Bradford (2008), uma prótese mioelétrica para membro superior, que foi 
o recurso mais recorrente nos artigos analisados no presente estudo, pode custar até mais que 30.000 dólares, o que pode inviabilizar a aquisição por grande parcela da população. Nos estudos sobre prótese mioelétrica, por exemplo, o custo variou entre $\$ 200,00$ e $\$ 1250,00$ dólares, apontando que esses materiais, aliados à impressão 3D, são capazes de viabilizar a aquisição por pessoas socioeconomicamente desfavorecidas.

Com relação aos limites da presente pesquisa, estes dizem respeito à ausência de padronização dos termos de pesquisa utilizados para a identificação do termo "impressão tridimensional", já que diversas palavras-chave são empregadas para se referir a esta, o que gerou dificuldade entre os pesquisadores durante a fase de coleta de dados. Além disso, nas diferentes bases de dados foram empregados cruzamentos com descritores diferentes, o que pode ter levado à perda de algum estudo. Sugere-se que futuras pesquisas sejam realizadas em outras bases de dados voltadas para as áreas de tecnologia, engenharia e design, além da área da saúde, e que incluam outras partes do corpo, além do membro superior, a fim de que mais estudos sejam localizados e a análise seja mais ampliada.

\section{Conclusáo}

Com base neste estudo, foi possível identificar que o uso da impressão 3D ocorreu com maior frequência para a confecção de próteses do tipo mioelétrica e com o público infantil. As órteses foram menos estudadas e as mais comuns encontradas foram as do tipo dinâmicas. Os principais materiais utilizados para a impressão dos dispositivos foram o PLA e o ABS. Identificou-se também que os estudos na área da confecção de órteses e próteses para membro superior a partir da impressão 3D, até este momento, encontram-se em fases iniciais de desenvolvimento e ainda não apresentam sua eficiência e eficácia com grandes grupos populacionais e/ou tipos de deficiências diversas. No entanto, os resultados iniciais se demonstraram relevantes devido ao custo-benefício dos produtos assistivos e da possibilidade de geração de modelos versáteis, o que nos sugere que é um campo promissor para ampliar e aprofundar a aplicação da impressão 3D como recurso facilitador no processo de reabilitação.

\section{Referências}

Agnelli, L. B., \& Toyoda, C. Y. (2003). Estudo de materiais para confecção de órteses e sua utilização prática por terapeutas ocupacionais no Brasil. Cadernos de Terapia Ocupacional da UFSCar, 11(2), 83-94.

Aguiar, L. C. D., \& Yonezawa, W. M. (2014). Construção de instrumentos didáticos com impressoras 3D. In Anais do 40 Simpósio Nacional de Ensino de Ciência e Tecnologia (SINECT). Ponta Grossa: UTFPR.

Athanasiou, K. A., Niederauer, G. G., \& Agrawal, C. M. (1996). Sterilization, toxicity, biocompatibility and clinical applications of polylactic acid/polyglycolic acid copolymers. Biomaterials, 17(2), 93-102.

Auras, R., Harte, B., \& Selke, S. (2004). An overview of polylactides as packaging materials. Macromolecular Bioscience, $4(9), 835-864$.

Baronio, G., Harran, S., \& Signoroni, A. (2016). A critical analysis of a hand orthosis reverse engineering and 3D printing process. Applied Bionics and Biomechanics, 2016, 1-7.

Bersch, R. (2017). Introdução à Tecnologia Assistiva. Recuperado em 4 de maio de 2019, de http://www.assistiva.com.br/Introducao_Tecnologia_Assistiva.pdf

Brasil. (2009). Tecnologia Assistiva. Brasília: CORDE.

Brasil. (2015a, 7 de julho). Lei no 13146, de 6 de julho de 2015. Institui a Lei Brasileira de Inclusão da Pessoa com Deficiência (Estatuto da Pessoa com Deficiência). Diário Oficial [da] República Federativa do Brasil, Brasília, seção 1, p. 72. 
Brasil. Conselho Federal de Fisioterapia e Terapia Ocupacional - COFFITO. (2015b). Resolução n 458, de 20 de novembro de 2015. Dispóe sobre o uso da Tecnologia Assistiva pelo terapeuta ocupacional e dá outras providências. Diário Oficial [da] República Federativa do Brasil, Brasília. Recuperado em 6 de abril de 2020, de https://www.coffito.gov.br/nsite/?p=3221

Cavalcanti, A., \& Galvão, C. (2011). Terapia ocupacional: fundamentação \& prática. Rio de Janeiro: Guanabara Koogan.

Cook, A. M., \& Polgar, J. M. (2015). Assistive Technologies: principles and practice. United States of America: Elsevier Mosby.

Costa, C. R., Ferreira, F. M. R. M., Bortolus, M. V., \& Carvalho, M. G. R. (2015). Dispositivos de tecnologia assistiva: fatores relacionados ao abandono. Cadernos de Terapia Ocupacional da UFSCar, 23(3), 611-624.

Diment, L. E., Thompson, M. S., \& Bergmann, J. H. M. (2018). Three-dimensional printed upper-limb prostheses lack randomised controlled trials: a systematic review. Prosthetics and Orthotics International, 42(1), 1-7.

Drumright, R. E., Gruber, P. R., \& Henton, D. E. (2000). Polylactic acid technology. Advanced Materials, 12(23), 1841-1846.

García-García, L. A., Rodríguez-Salvador, M., \& Moya-Bencomo, M. D. (2018). Development of a customized wrist orthosis for flexion and extension treatment using reverse engineering and 3D printing. In Annals World Congress on Medical Physics and Biomedical Engineering (pp. 609-613). Singapore: Springer.

Gerstle, T. L., Ibrahim, A. M. S., Kim, P. S., Lee, B. T., \& Lin, S. J. (2014). A plastic surgery application in evolution: three-dimensional printing. Plastic and Reconstructive Surgery, 133(2), 446-451.

Gonçalves, B. A., \& Francisco, N. P. F. (2011). Órteses: orientaçôes e cuidados. In Anais $14^{\circ}$ Encontro Latino Americano de Iniciação Científica e $10^{\circ}$ Encontro Latino Americano de Pós-Graduação-Universidade do Vale do Paraíba. São José dos Campos: UNIVAP.

Gretsch, K. F., Lather, H. D., Peddada, K. V., Deeken, C. R., Wall, L. B., \& Goldfarb, C. A. (2016). Development of novel 3D-printed robotic prosthetic for transradial amputees. Prosthetics and Orthotics International, 40(3), 400-403.

Guerra Neto, C. L. B., Nagem, D. A. P., Hékis, H. R., Coutinho, K. D., \& Valentim, R. A. M. (2018). Tecnologia 3D na saúde: uma visão sobre órteses e próteses, tecnologias assistivas e modelagem 3D. Natal: SEDIS/UFRN.

International Organization for Standardization - ISO. (1989). ISO 8549-1:1989. Prosthetics and orthotics Vocabulary - Part 1: General terms for external limb prostheses and external orthoses. Recuperado em 29 de junho de 2019, de https://www.iso.org/obp/ui/\#iso:std:iso:8549:-1:ed-1:v1:en

Krebs, D. E., Edelstein, J. E., \& Thornby, M. A. (1991). Prosthetic management of children with limb deficiencies. Physical Therapy, 71(12), 920-934.

Maia, B. A. (2016). Parametrização dimensional, por modelo de regressão, de próteses de mão para crianças, confeccionadas por manufatura aditiva (Dissertação de mestrado). Universidade Federal de Goiás, Catalão.

Mallmann, T. S. (2018). O uso de impressão 3D no auxílio às pessoas usuárias de órteses: um projeto de design focado em tecnologia assistiva (Monografia). Universidade do Vale do Taquari, Lajeado.

Martins, E. P. (2018). Modelagem da Resposta de Mecanorreceptores Táteis SAII com Circuitos MOS (Trabalho de Conclusão de Curso). Universidade Federal de Santa Maria, Santa Maria.

McGimpsey, G., \& Bradford, T. C. (2008). Limb prosthetics services and devices. Worcester: Bioengineering Institute Center for Neuroprosthetics.

Merchant, R., Cruz, D., Ballesteros, M., \& Chairez, I. (2018). Integrated wearable and self-carrying active upper limb orthosis. Proceedings of the Institution of Mechanical Engineers. Part H, Journal of Engineering in Medicine, 232(2), 172-184.

Nedel, W. L., \& Silveira, F. (2016). Os diferentes delineamentos de pesquisa e suas particularidades na terapia intensiva. Revista Brasileira de Terapia Intensiva, 28(3), 256-260.

Paschoarelli, L. C., \& Menezes, M. S. (2009). Design e ergonomia: aspectos tecnológicos. São Paulo: Cultura Acadêmica.

Pereira, H. (2016). Prótese mioelétrica para membro superior implementada em FPGA (Trabalho de Conclusão de Curso). Universidade Federal de Santa Catarina, Araranguá.

Polis, J. E. (2009). Projeto e construção de parte estrutural de prótese de mão humana com movimentos (Dissertação de mestrado). Universidade Estadual de Campinas, Campinas. 
Radomski, M. V., \& Latham, C. A. T. (2013). Terapia ocupacional para disfunçôes físicas. São Paulo: Santos.

Rodrigues Júnior, J. L. R., Cruz, L. M. S., \& Sarmanho, A. P. S. (2018). Impressora 3D no desenvolvimento de pesquisas com próteses. Revista Interinstitucional Brasileira de Terapia Ocupacional, 2(2), 398-413.

Silva, L. A., Medola, F. O., Rodrigues, O. V., Rodrigues, A. C. T., \& Sandnes, F. E. (2018). Interdisciplinary-based development of user-friendly customized 3D printed upper limb prosthesis. In Annals 9th International Conference on Applied Human Factors and Ergonomics (pp. 899-908). Florida: AHFE.

Souza, M. T., Silva, M. D., \& Carvalho, R. (2010). Revisão integrativa: o que é e como fazer. Einstein, 1(1), 102-106.

Vink, E. T. H., Rábago, K. R., Glassner, D. A., \& Gruber, P. R. (2003). Applications of life cycle assessment to NatureWorks ${ }^{\mathrm{TM}}$ polylactide (PLA) production. Polymer Degradation \& Stability, 80(3), 403-419.

Wagner, J. B., Scheinfeld, L., Leeman, B., Pardini, K., Saragossi, J., \& Flood, K. (2018). Three professions come together for an interdisciplinary approach to 3D printing: occupational therapy, biomedical engineering, and medical librarianship. Journal of the Medical Library Association: JMLA, 106(3), 370-376.

Xu, G., Gao, L., Tao, K., Wan, S., Lin, Y., Xiong, A., Kang, B., \& Zeng, H. (2017). Three-dimensional-printed upper limb prosthesis for a child with traumatic amputation of right wrist: a case report. Medicine, 96(52), 1-5.

Yoshikawa, M., Sato, R., Higashihara, T., Ogasawara, T., \& Kawashima, N. (2015). Rehand: realistic electric prosthetic hand created with a 3D printer. In Annals 37th Annual International Conference of the IEEE Engineering in Medicine and Biology Society - EMBC (pp. 2470-2473). USA: IEEE.

Yoshikawa, M., Taguchi, Y., Sakamoto, S., Yamanaka, S., Matsumoto, Y., Ogasawara, T., \& Kawashima, N. (2013). Trans-radial prosthesis with three opposed fingers. In Annals International Conference on Intelligent Robots and Systems (pp. 1493-1498). USA: IEEE.

Zuniga, J. M., Carson, A. M., Peck, J. M., Kalina, T., Srivastava, R. M., \& Peck, K. (2017). The development of a low-cost three-dimensional printed shoulder, arm, and hand prostheses for children. Prosthetics and Orthotics International, 41(2), 205-209.

Zuniga, J., Katsavelis, D., Peck, J., Stollberg, J., Petrykowlski, M., Carson, A., \& Fernandez, C. (2015). Cyborg beast: a low-cost 3D-printed prosthetic hand for children with upper-limb differences. BMC Research Notes, 8(10), 1-8.

\section{Contribuiçáo dos Autores}

Sandra Yoshie Uraga Morimoto: Concepção de texto; organização dos instrumentos para coleta de dados; coleta, tabulação e análise dos dados; redação do artigo. Ana Karina Pessoa da Silva Cabral, Danielle Carneiro de Menezes Sanguinetti, José Ângelo Peixoto da Costa, Weldma Karlla Coelho, Giselle Schimidt Alves Diaz Merino: Análise dos dados e revisão do texto. Elvis da Silva Rodrigues de Freitas: Coleta, tabulação e análise dos dados. Daniela Salgado Amaral: Análise dos dados, revisão do texto e orientação da pesquisa. Todos os autores aprovaram a versão final do texto.

\section{Autor para correspondência}

Sandra Yoshie Uraga Morimoto e-mail: sandrap_ucca@hotmail.com, sandra.syum@gmail.com

\section{Editora de seçáo}

Marcia Maria Pires Camargo Novelli 